\title{
Overexpression of Tumour Necrosis Factor- $\alpha$ - Induced Protein 8 is Associated with Prognosis in Colon Cancer
}

\section{Xingqi Zhang \\ Zequn Li \\ Yuqi Sun \\ Gan Liu (D) \\ Xiaodong Liu \\ Yanbing Zhou (1D)}

Department of Gastrointestinal Surgery, Affiliated Hospital of Qingdao University, Qingdao, Shandong, People's Republic of China
Correspondence: Yanbing Zhou

Department of Gastrointestinal Surgery, Affiliated Hospital of Qingdao University, 16\# Jiangsu Road, Qingdao, Shandong, People's Republic of China

Email zhouyanbing999@aliyun.com
Purpose: The present study aimed to examine the association of tumour necrosis factor- $\alpha$ induced protein 8 (TIPE) expression levels with clinicopathological features and prognosis of patients with colon cancer following surgery.

Patients and Methods: The present study included 200 patients with colon cancer who underwent colon resection between June 2011 and October 2012. All follow-ups were censored in July 2020, with a median follow-up time of 62.25 months. Kaplan-Meier survival curve analysis and Cox regression analysis were used to determine predictors for the overall survival rate.

Results: High expression of TIPE was associated with lymph node metastasis, higher Dukes' stage and right-sided colon cancer (RCC). An exploratory subgroup analysis found that high expression of TIPE was associated with age $\geq 65$, lymphatic invasion and higher Dukes' stage only in the RCC group $(P<0.05)$, whereas no similar trend was observed in the left-sided colon cancer (LCC) subgroup. Age $\geq 65$, differentiation, lymph node metastasis and TIPE expression levels were independent prognostic factors influencing the survival rate of patients with colon cancer following surgery in multivariate Cox analysis $(P<0.05)$. ROC curve analysis showed that the immunoreactive score of TIPE had good predictive value for five-year survival rates $(\mathrm{AUC}=0.727)$ and lymph node metastasis $(\mathrm{AUC}=0.760)$ among patients with RCC. Survival analysis revealed that the expression of TIPE had a significant impact on survival, and higher expression levels suggested a worse prognosis.

Conclusion: This study demonstrated that TIPE may be a novel biomarker for predicting the survival outcome and lymph node metastasis. TIPE was overexpressed in colon cancer tissue and significantly associated with poor patient survival, especially in patients with RCC.

Keywords: colonic neoplasms, TIPE, biomarker, prognosis, immunohistochemistry

\section{Introduction}

Colon cancer, one of the most common malignancies worldwide and one of the most common causes of cancer deaths, represents a major public health problem. ${ }^{1}$ Although colon cancer is highly treatable in its early stage, it is the second leading cause of cancer-related deaths in Western countries and the fourth leading cause of cancer-related deaths in China. ${ }^{2-4}$ Colon cancer is generally a slow process spanning decades from cancer initiation to diagnosis and theoretically, provides considerable opportunity for early detection. Unfortunately, only $39 \%$ of patients are diagnosed at an early stage of the disease when the 5 -year survival rate is $90 \%{ }^{5}$ 
Given the important survival implications, there is an emerging interest in identifying colon cancer in the early stage of the disease to improve the cure rate of advanced colon cancer.

There are clinically distinct prognoses and treatment outcomes between right-sided colon cancer (RCC) and left-sided colon cancer (LCC), although colon cancer is usually classified as a single disease. ${ }^{6}$ Previous studies have identified the differences in molecular biology, pathology, prognosis and epidemiology between LCC and RCC. ${ }^{7-10}$ The poorer biology (higher-grade tumours, more lymphovascular invasion, higher proportion of gene mutations) of RCC worsened the prognosis of patients who already had nodal and distant metastases. ${ }^{11}$ The difference in anatomic structure and oncogenic alterations may partly explain the different clinical presentations of RCC and LCC, such as more advanced T stage with severe symptoms (passage trouble or abdominal mass) and more mutations in RCC patients than in LCC patients. ${ }^{12,13}$ In contrast, several studies did not identify an association between tumour location within the colon and the overall survival time. ${ }^{14}$

Tumour necrosis factor- $\alpha$-induced protein 8 (TIPE) also known as TNFAIP8, SCC-S2, GG2-1 or NDED is a recently identified protein that has been reported to promote cell proliferation, angiogenesis, invasion, metastasis, drug resistance, autophagy, and tumorigenesis by inhibiting cell apoptosis in human cancers. ${ }^{15,16}$ TIPE is the founding member of the TIPE -family which also includes the TNFAIP8-like 1 (TIPE1), TNFAIP8-like 2 (TIPE2), and TNFAIP8-like 3 (TIPE3) proteins and plays important roles in the development and progression of cancers. ${ }^{17-23}$ Overexpression of TIPE is frequently observed in malignant cells and is strongly linked to excessive proliferation, reduced apoptosis, enhanced invasion and metastasis. ${ }^{24}$ Studies have shown that TIPE overexpression was correlated with cancer progression and poor prognosis in patients with non-small cell lung cancer. ${ }^{24}$ However, no research has shown whether there is a correlation between the overexpression of TIPE and the prognosis of patients with colon cancer, especially patients with left or right-sided colon cancer.

Therefore, the aim of the present study was to investigate the expression of TIPE in left- and right-sided colon cancer tissue samples and evaluate its prognostic significance by analyzing the association between the level of TIPE expression and survival time outcomes.

\section{Patients and Methods}

\section{Patients and Clinical Tissue Samples}

The present study included patients who underwent colon cancer resection from June 2011 to April 2012. Patients with rectal cancer were excluded, as were patients without an explicit tumour location or lymph node count recorded in the pathology report. Patients who received neoadjuvant therapy were excluded to avoid immune changes and inflammatory reactions caused by radiotherapy and chemotherapy interfering with the results. Only one patient with distant metastasis of colon cancer was excluded. Two hundred cancerous tissues and matched adjacent tissues were obtained from enrolled patients. The study population consisted of 92 male and 108 female patients. The tumour stage was determined according to the 2016 World Health Organization Classification of Tumours of the Digestive System, the American Joint Committee on Cancer (AJCC)/Union for International Cancer Control (UICC) tumour node metastasis (TNM) system and the modified Dukes' criteria classification. ${ }^{25,26}$ The clinical features of the patients and the macroscopic features of the tumours were obtained from the hospital's electronic record system. Overall survival (OS) was defined as the time from primary resection to death from cancer. The follow-up period was considered to end in July 2020 or at the time of death of the patient. Following surgical removal, the tumour biopsy samples were formalin-fixed $(10 \%)$ for $24 \mathrm{~h}$ at $4^{\circ} \mathrm{C}$, paraffin-embedded and stored at $4^{\circ}$ $\mathrm{C}$ for further histological analysis and immunohistological studies. The present study followed the Declaration of Helsinki and was approved by the Ethics Committee of the Affiliated Hospital of Qingdao University. Written informed consent was obtained from each patient.

Surgically excised tumour specimens were fixed with $10 \%$ neutral formalin and embedded in paraffin, and $4-\mu \mathrm{m}$-thick sections were prepared. Antigen retrieval was performed by heating the tissue sections at $95^{\circ} \mathrm{C}$ in 0.01 $\mathrm{M}$ citrate buffer solution, $\mathrm{pH} 6.0$, for $5 \mathrm{~min}$ in a stainless steel pressure cooker. Hydrogen peroxide $(0.3 \%)$ was applied to block endogenous peroxide activity, and the sections were incubated with normal goat serum to reduce nonspecific binding. The tissue sections were incubated with TIPE rabbit polyclonal antibody (1:200 dilution; Abcam, USA), and rabbit immunoglobulin was used as a negative control. The specimens were immunostained using a custom-made and validated anti-TIPE antibody (1:50; BOSTER, Wuhan, China) according to the 
manufacturer's directions. Haematoxylin was used for counterstaining for $1 \mathrm{~min}$ at room temperature.

\section{Evaluation of Immunohistochemical Staining}

Surgically excised tumour specimens were fixed with $10 \%$ neutral formalin and embedded in paraffin, and 4- $\mu$ m-thick sections were prepared. Antigen retrieval was performed by heating the tissue sections at $95^{\circ} \mathrm{C}$ in $0.01 \mathrm{M}$ citrate buffer solution, $\mathrm{pH} 6.0$, for $5 \mathrm{~min}$ in a stainless steel pressure cooker. Hydrogen peroxide $(0.3 \%)$ was applied to block endogenous peroxide activity, and the sections were incubated with normal goat serum to reduce nonspecific binding. The tissue sections were incubated with TIPE rabbit polyclonal antibody (1:200 dilution; Abcam, USA), and rabbit immunoglobulin was used as a negative control. The specimens were immunostained using a custom-made and validated anti-TIPE antibody (1:50; BOSTER, Wuhan, China) according to the manufacturer's directions. Haematoxylin was used for counterstaining for $1 \mathrm{~min}$ at room temperature.

\section{Statistical Analysis}

Statistical analysis was performed using SPSS 24.0 statistical software (SPSS, IL, USA) and GraphPad Prism 8.0.2. The collinearity of predictor variables was assessed using the "usdm" package in R (version 3.5.3.). Patient characteristics were grouped by the IRS of TIPE, with comparisons made using the Mann-Whitney $U$ and Pearson chisquare tests for continuous and categorical variables, respectively. OS was summarised by IRS of TIPE using standard Kaplan-Meier methods, where estimates of the median OS and 5-year OS rates were obtained. Receiver operating characteristic (ROC) curves and the area under the ROC curve (AUC) were established to evaluate the diagnostic value of the IRS for TIPE. A value of $P<0.05$ was considered to be statistically significant.

\section{Results}

\section{Clinical Characteristics}

The clinical and pathological characteristics of 200 patients with colon cancer are presented in Table 1. A total of 200 patients were enrolled in this study, including 108 females $(54.0 \%)$ and 92 males (46.0\%), and the mean age was $62.25 \pm 11.03$ years (range 33-91 years). According to the revised Dukes' staging, there were 11 patients with Duke's A colon cancer, 110 patients with
Duke's B colon cancer, 79 patients with Duke's C colon cancer and no patients with Duke's D colon cancer because of the exclusion criteria. All patients were closely followed after surgery, with a median follow-up period of 62.25 months (range, 2-113 months) and 87.02 months for the surviving patients (range, 63-113 months). As described in Table 1, the tumour was located in the left colon (distal transverse colon, descending colon, or sigmoid) or the right colon (transverse, proximal transverse colon, or caecum) in 89 (44.5\%) and 111 (55.5\%) patients, respectively. Analysis based on the WHO histologic subtypes revealed that $15(7.5 \%)$ tumours were welldifferentiated tubular adenocarcinoma (TAC), 149 (74.5\%) tumours were moderately differentiated TAC, and $36(18.0 \%)$ tumours were poorly differentiated TAC, mucinous carcinoma or signet ring cell carcinomas.

Staining for TIPE showed immunoreactivity in the nucleoplasm in both LCC and RCC tissues compared with adjacent normal tissues (Figure 1A-F). A semiquantitative score was assigned based on the TIPE immunostaining activity according to the intensity and area of the stained cells, as described in the text. The IRS, which represented the TIPE expression level in colon cancer tissues and adjacent normal tissue, was calculated for comparisons among the diagnostic cohort. Overexpressed TIPE was detected in 200 colon cancer samples, whereas negative or weak TIPE immunoreactivity was observed in adjacent normal tissue (IRS in colon cancer samples, $4.00 \pm 2.40$; IRS in adjacent normal tissues, $2.55 \pm 1.61, \quad P<0.0001$, Figure 1G). Additionally, the expression of TIPE protein was found to be significantly higher in colon cancer patients with RCC (IRS, 4.45 \pm 2.42 ) and lymph node metastasis (IRS, 4.95 \pm 2.38 ) than in those with LCC (IRS, $3.43 \pm 2.26, P<0.001$, Figure 1H) and without lymph node metastasis (IRS, 3.37 \pm 2.23 , Figure 1I). The baseline characteristics of the two groups are shown in Table 2. The clinical data included sex, age, differentiation, depth of invasion, lymph node metastasis, Dukes' stage, location, and histological type. There were significant differences in TIPE expression by age, lymph node invasion and Dukes' stage $(P<0.05)$. No other significant differences were observed. These results indicate that positive correlations exist between TIPE and certain clinicopathological features of patients with colon cancer, namely, the location of tumorigenesis, Dukes' stage and lymph node metastasis. 
Table I Associations of TIPE Expression with Various Clinicopathological Factors of Colon Cancer Patients

\begin{tabular}{|c|c|c|c|c|c|}
\hline \multirow[t]{2}{*}{ Characteristics } & \multirow[t]{2}{*}{$\mathbf{n}$} & \multicolumn{2}{|c|}{ TIPE Expression } & \multirow[t]{2}{*}{$X^{2}$} & \multirow[t]{2}{*}{$P$-value } \\
\hline & & High (\%) & Low (\%) & & \\
\hline Total & 200 & 64 & 136 & & \\
\hline Follow-up & & $2-113$ months & & & \\
\hline Survival time & & $46.88 \pm 34.87$ & $69.49 \pm 30.50$ & & \\
\hline Age (years) & & & & 0.641 & 0.423 \\
\hline$\leq 65$ & 102 & 30 (46.88\%) & 72 (52.94\%) & & \\
\hline$>65$ & 98 & $34(53.13 \%)$ & 64 (47.06\%) & & \\
\hline Gender & & & & 0.787 & 0.375 \\
\hline Male & 92 & 31 (48.44\%) & 61 (44.85\%) & & \\
\hline Female & 108 & 33 (5I.56\%) & 75 (55.15\%) & & \\
\hline Differentiation & & & & 0.146 & 0.965 \\
\hline Well & 15 & $5(7.81 \%)$ & $10(7.35 \%)$ & & \\
\hline Moderate & 149 & 47 (73.44\%) & $102(75.00 \%)$ & & \\
\hline Poor & 36 & $12(18.75 \%)$ & $24(17.65 \%)$ & & \\
\hline Lymphatic invasion & & & & 18.100 & $<0.001$ \\
\hline No & 121 & 25 (39.06\%) & 96 (70.59\%) & & \\
\hline Yes & 79 & 39 (60.94\%) & 40 (29.4I\%) & & \\
\hline Tumor stage & & & & 0.508 & 0.257 \\
\hline I+II & 11 & $2(3.13 \%)$ & 9 (6.62\%) & & \\
\hline III+IV & 189 & $62(96.88 \%)$ & 127 (93.38\%) & & \\
\hline Dukes' stage & & & & 17.588 & $<0.001$ \\
\hline Dukes' A & 11 & $2(3.13 \%)$ & 9 (6.62\%) & & \\
\hline Dukes' B & 110 & $23(35.94 \%)$ & 87 (63.97\%) & & \\
\hline Dukes' C & 79 & 39 (60.94\%) & 40 (29.4l\%) & & \\
\hline Location & & & & 8.361 & 0.004 \\
\hline Left & 89 & 19 (29.69\%) & 70 (5I.47\%) & & \\
\hline Right & 111 & 45 (70.3।\%) & 66 (48.53\%) & & \\
\hline Histological type & & & & 0.032 & 0.857 \\
\hline$M C$ & 33 & II (17.19\%) & $22(16.18 \%)$ & & \\
\hline NMC & 167 & 53 (82.8I\%) & II 4 (83.82\%) & & \\
\hline
\end{tabular}

Abbreviations: MC, mucinous adenocarcinoma; NMC, non-mucinous adenocarcinoma.

\section{Association of TIPE Expression with Clinical Characteristics}

The optimum cut-off value for the IRS to assess statistical significance was selected based on the association with the patients' OS by using X-tile plots (IRS=5.5). The 200 patients with colon cancer were divided into the high or low expression group according to the best cutoff point of TIPE expression level in the diagnostic cohort. $68.0 \%(136 / 200)$ of the patients had low TIPE expression, and $32.0 \%(64 / 200)$ had high TIPE expression. The baseline characteristics of the two groups are shown in Table 1. The clinical data included sex, age, differentiation, depth of invasion, lymph node metastasis, Dukes' stage, location, and histological type. There were significant differences in TIPE expression in the presence or absence of lymph node metastasis, Dukes' stage and location $(P<0.05)$. No other significant differences were observed. These results indicate that positive correlations exist between TIPE expression and certain clinicopathological features of patients with colon cancer, namely, the location of tumorigenesis, Dukes' stage and lymph node metastasis. 
A

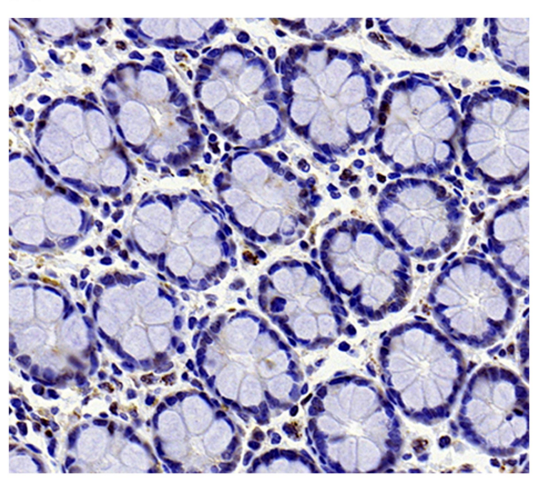

D

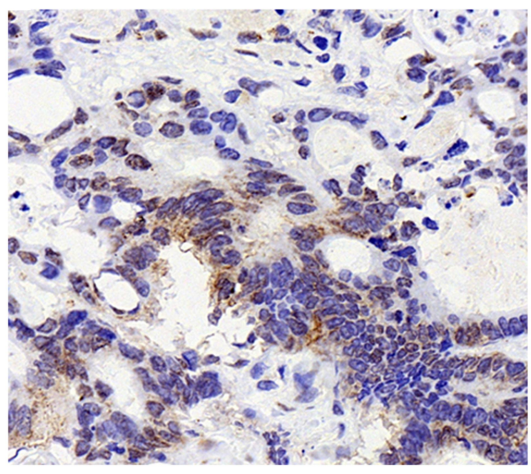

G

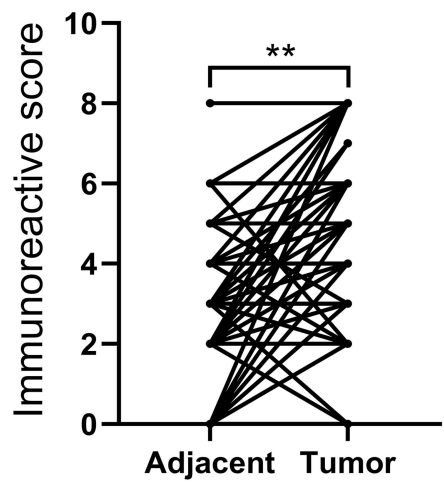

B

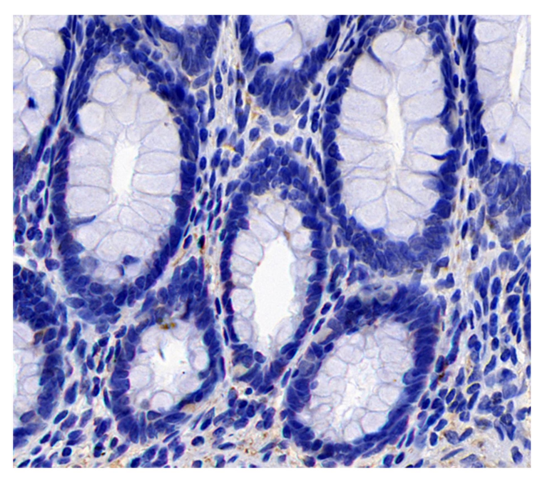

E

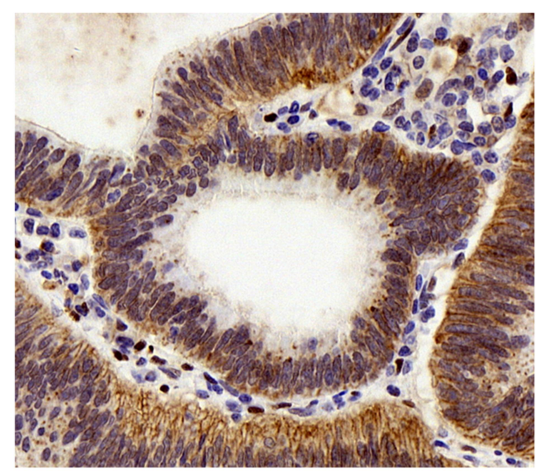

H

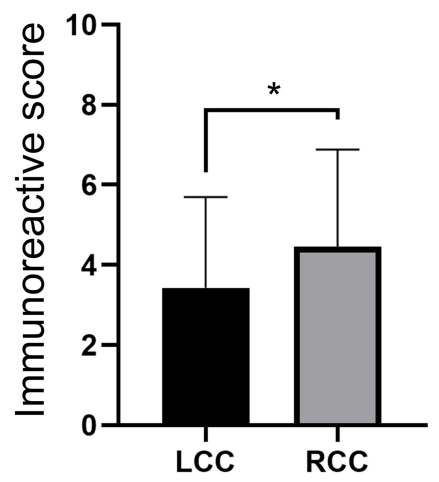

C

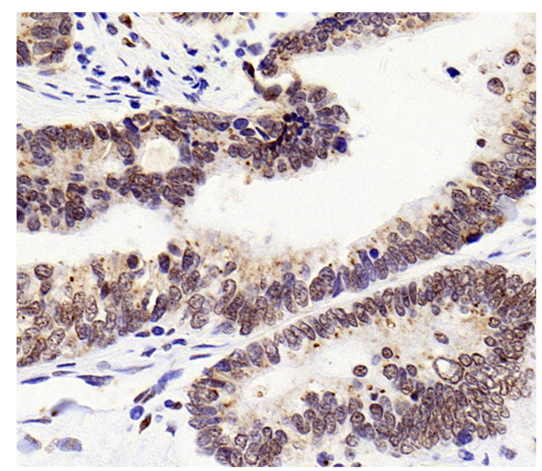

F

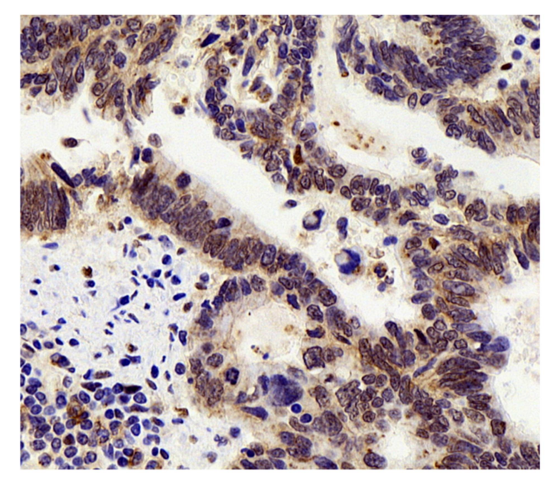

I

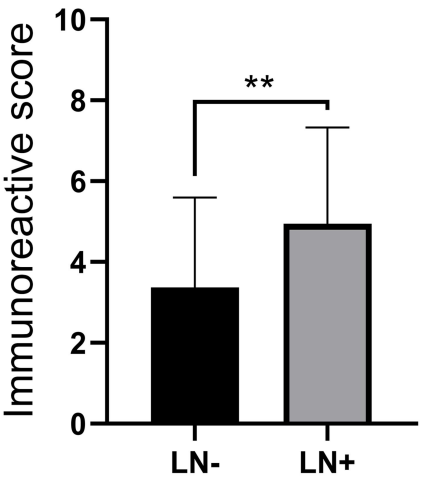

Figure I The expression of TIPE in different samples. (A) Representative immunohistochemical staining showed low TIPE expression in left-side adjacent normal tissue. (B) Representative immunohistochemical staining showed low TIPE expression in right-side adjacent normal tissue. (C) Expression of TIPE in tumor tissues without lymphatic invasion. (D) Representative immunohistochemical staining showed high TIPE expression in left-side colon cancer. (E) Representative immunohistochemical staining showed high TIPE expression in right-side colon cancer. (F) Expression of TIPE in tumor tissues with lymphatic invasion. (G) Before-after plot showing the immunoreactive score (IRS) of paired tumor tissue and adjacent normal tissue for TIPE. (H) The IRS of TIPE expression in LCC and RCC. (I) IRS of TIPE expression in colon cancer specimens with or without lymph node metastasis. $* P<0.01$; * $* P<0.0001$.

\section{Receiver Operating Characteristics Curve Analysis}

We performed ROC curve analysis to determine the predictive value of TIPE expression for survival rate and lymph node metastasis. As shown in Figure 2A, the ROC curve revealed that the IRS was able to predict the five-year survival rates of patients with non-metastatic colon cancer (Table 3, AUC =
0.645). In the subgroup analysis, ROC curve analysis showed that the IRS had good predictive accuracy for the prognosis of RCC (Table 3 and Figure 2B, AUC $=0.727$ ). However, the ROC curve analysis did not show good diagnostic value for LCC with an AUC of 0.516 (Table 3 and Figure 2C). To further explore whether TIPE could serve as a biomarker in predicting lymph node metastasis, ROC curve was constructed and AUC 
Table 2 Associations of TIPE Expression with Various Clinicopathological Factors of Right-Sided and Left-Sided Colon Cancer Patients

\begin{tabular}{|c|c|c|c|c|c|c|}
\hline \multirow[t]{2}{*}{ Characteristics } & \multicolumn{2}{|c|}{ RCC (\%) } & \multirow[t]{2}{*}{ P-value } & \multicolumn{2}{|c|}{ LCC (\%) } & \multirow[t]{2}{*}{ P-value } \\
\hline & Low & High & & Low & High & \\
\hline Total & 66 & 45 & & 70 & 19 & \\
\hline Age, years: & & & 0.028 & & & 0.617 \\
\hline$<60$ & 36 & 19 & & 36 & 11 & \\
\hline$\geq 60$ & 30 & 36 & & 34 & 8 & \\
\hline Gender: & & & 0.730 & & & 0.324 \\
\hline Male & 33 & 21 & & 28 & 10 & \\
\hline Female & 33 & 24 & & 42 & 9 & \\
\hline Differentiation & & & 0.575 & & & 0.203 \\
\hline Well & 6 & 2 & & 4 & 3 & \\
\hline Moderate & 48 & 32 & & 54 & 15 & \\
\hline Poor & 12 & 11 & & 12 & 1 & \\
\hline Lymphatic invasion & & & $<0.001$ & & & 0.131 \\
\hline No & 53 & 17 & & 43 & 8 & \\
\hline Yes & 13 & 28 & & 27 & 11 & \\
\hline Tumor stage & & & 1.000 & & & 0.580 \\
\hline $1+11$ & 4 & 2 & & 5 & 0 & \\
\hline III+IV & 62 & 43 & & 65 & 19 & \\
\hline Dukes' stage & & & 0.001 & & & 0.260 \\
\hline Dukes' A & 4 & 2 & & 5 & 0 & \\
\hline Dukes' B & 49 & 15 & & 38 & 8 & \\
\hline Dukes' C & 13 & 28 & & 27 & 11 & \\
\hline Histological type & & & 0.969 & & & 0.784 \\
\hline MC & 13 & 9 & & 9 & 2 & \\
\hline NMC & 53 & 36 & & 61 & 17 & \\
\hline
\end{tabular}

Abbreviations: RCC, right-sided colon cancer; LCC, left-sided colon cancer; MC, mucinous adenocarcinoma; NMC, non-mucinous adenocarcinoma.

was calculated. In Figure 2D, ROC curve showing the capability of TIPE in distinguishing patients with lymph node metastasis with an AUC of 0.683 (Table 4). Figure 2E showed that the IRS had good predictive accuracy for the prognosis of RCC with an AUC of 0.760 (Table 4). On ROC analysis in LCC, the AUC for IRS was 0.637 (Table 4 and Figure 2F).

\section{Correlation Between TIPE \& Clinical Prognosis}

The median overall survival time of the enrolled patients in the present study was 62.25 months (range, 2 113 months). The three- and five-year disease-specific survival rates were $69.5 \%$ and $61.5 \%$, respectively. The OS curves were generated by Kaplan-Meier analysis. The results clarified that the mean OS time for colon cancer patients expressing low levels of TIPE was 69.49 months, and the 5-year OS rate was 71.3\%; the mean OS time for patients with colon cancer with high levels of TIPE was 46.88 months, and the 5-year OS rate was 40.6\% $(P<0.001$, Figure $3 \mathrm{~A}-\mathrm{D})$. Univariate and multivariate analyses using the Cox proportional hazards regression model were performed to further investigate factors associated with the prognosis of patients. We omitted Dukes' stage from the multiple regression analyses because of collinearity between Dukes' stage and lymphatic invasion. As shown in Table 5, univariate survival analysis revealed that OS was significantly associated with age, differentiation, lymph node metastasis and TIPE expression levels $(P<0.001)$. The rate of lymph node metastasis in the high TIPE expression group was significantly higher than that in the low TIPE expression group (60.9\% vs $29.4 \%$ ). Further multivariate analyses were performed to screen for independent prognostic factors. Age, differentiation, lymph node metastasis and TIPE expression levels were independent prognostic factors in the multivariate analysis.

\section{Discussion}

Recently, various investigations have established that gene expression changes are directly associated with the development of gastro-enteric tumours, such as colon cancer. The TIPE family is a newly described regulator of immunity and tumorigenesis. TIPE was the first novel protein discovered in this family and acts as an antiapoptotic and oncogenic molecule. ${ }^{27,28}$ High expression of TIPE has recently been found in several malignancies and tumour cells. Studies have found that TIPE is highly expressed in pancreatic cancer, gastric cancer, endometrial cancer and other tumour cells, regulating the growth, proliferation, migration and invasion of tumour cells. ${ }^{29-31}$ The TIPE protein was found to be significantly overexpressed in colon cancer tissues compared with tumour-adjacent tissues and was associated with the degree of malignancy of the tumour. ${ }^{32}$ Zhong et al detected the relative mRNA expression levels of TIPE family members in tumour and adjacent tissue samples of patients with colorectal cancer. The mRNA expression level of TIPE family members in colorectal cancer tissues was significantly lower than that in adjacent tissues. However, when the protein expression level of TIPE family members in 5 patients with colon cancer was detected by Western blot analysis, the TIPE protein expression was only partially consistent with its mRNA expression level. ${ }^{33}$ Their study is also in agreement with the previous finding by Lowe et. ${ }^{34}$ We examined 200 pairs of patient samples by immunostaining to further 
A
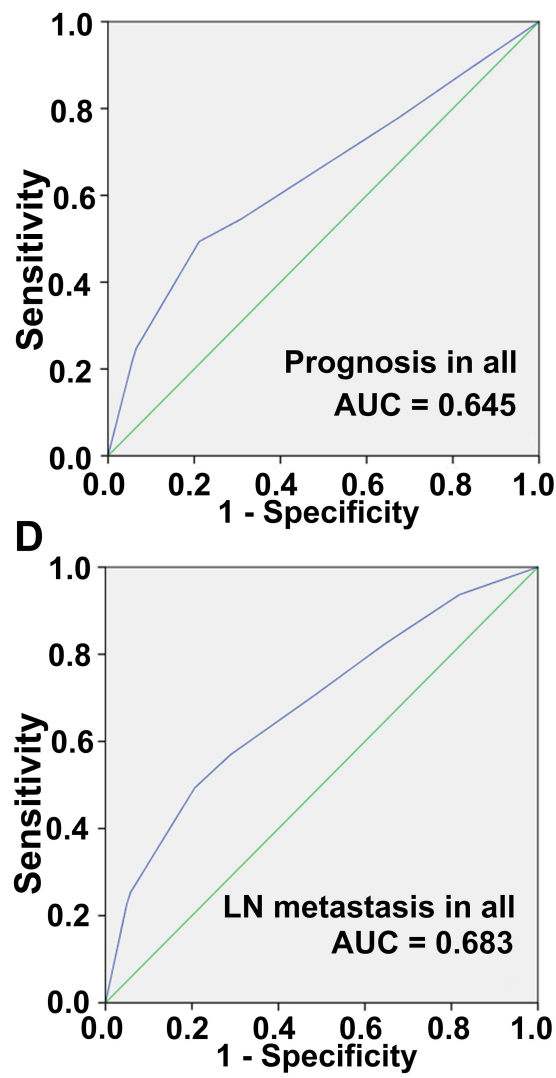

B

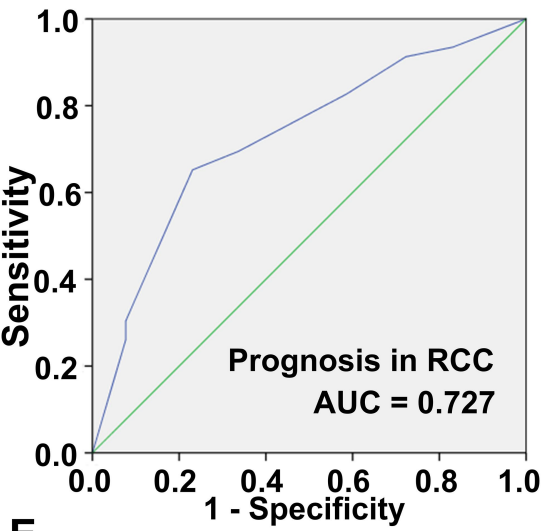

$E$

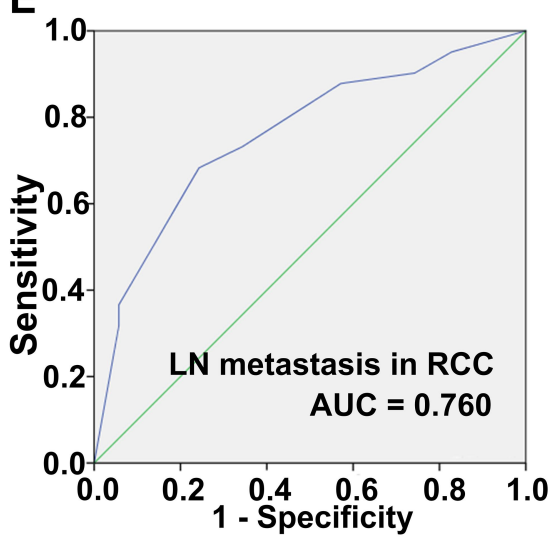

C

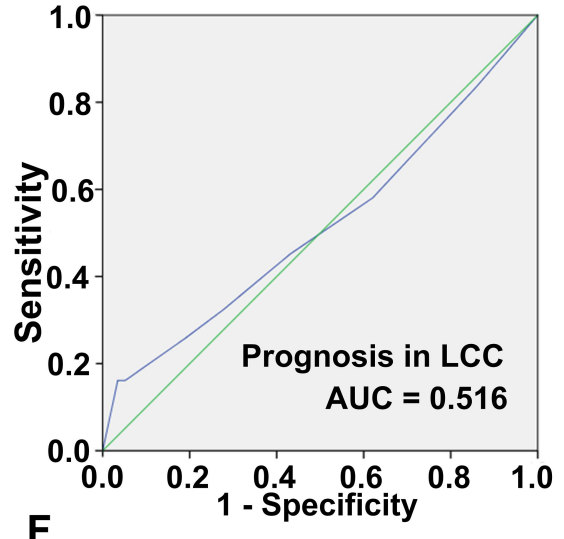

$\mathbf{F}$

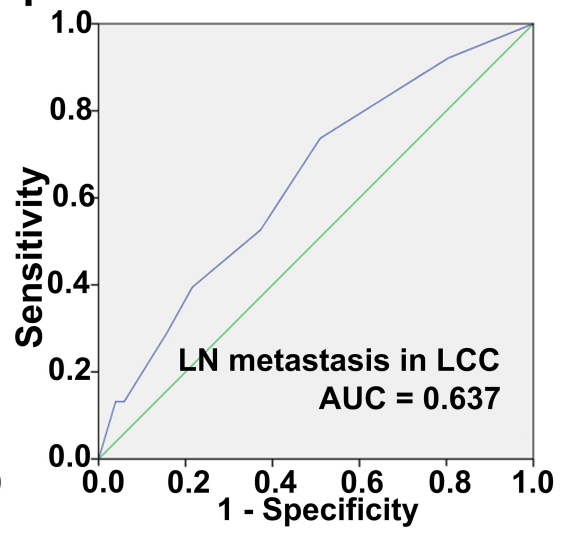

Figure 2 ROC curves of the prediction index value in predicting five-year overall survival of patients with (A) colon cancer; (B) right-sided colon cancer; (C) left-sided colon cancer. ROC curves of the prediction index value in predicting lymph node metastasis of patients with (D) colon cancer; (E) right-sided colon cancer; (F) left-sided colon cancer.

explore TIPE expression in cancer. The results demonstrated that the expression level of TIPE was higher in colon cancer tissues than in paracancerous tissues. The TIPE expression may be affected by multiple signaling pathways and closely related to tumorigenesis and tumour development. However, the relationship between TIPE expression and clinicopathological features and long-term survival of colon cancer has not been fully elucidated.

The present study enrolled 200 patients with primary colon cancer who underwent radical surgery and complete

Table 3 ROC-Related Parameters for Predicting 5-Year Overall Survival

\begin{tabular}{|l|l|l|l|l|l|l|}
\hline Groups & AUC & $\mathbf{9 5 \%} \mathbf{~ C l}$ & Specificity (\%) & Sensitivity (\%) & Youden Index (\%) & Cut-off value \\
\hline All patients & 0.645 & $0.563-0.726$ & 49.4 & 78.9 & 0.283 & 5.5 \\
RCC & 0.727 & $0.630-0.825$ & 65.2 & 76.9 & 0.421 & 5.5 \\
LCC & 0.516 & $0.383-0.648$ & 16.1 & 99.6 & 0.127 & 7.5 \\
\hline
\end{tabular}

Abbreviations: ROC, receiver operating characteristic; AUC, area under the curve; Cl, confidence interval; RCC, right-sided colon cancer; LCC, left-sided colon cancer.

Table 4 ROC-Related Parameters for Predicting Lymph Node Metastasis

\begin{tabular}{|l|l|l|l|l|l|l|}
\hline Groups & AUC & $\mathbf{9 5 \%} \mathbf{C l}$ & Specificity (\%) & Sensitivity (\%) & Youden Index (\%) & Cut-off value \\
\hline All patients & 0.683 & $0.607-0.759$ & 49.4 & 79.3 & 0.287 & 5.5 \\
RCC & 0.760 & $0.665-0.856$ & 68.3 & 75.7 & 0.440 & 5.5 \\
LCC & 0.637 & $0.521-0.753$ & 39.5 & 78.4 & 0.179 & 4.5 \\
\hline
\end{tabular}

Abbreviations: ROC, receiver operating characteristic; AUC, area under the curve; Cl, confidence interval; RCC, right-sided colon cancer; LCC, left-sided colon cancer. 
A

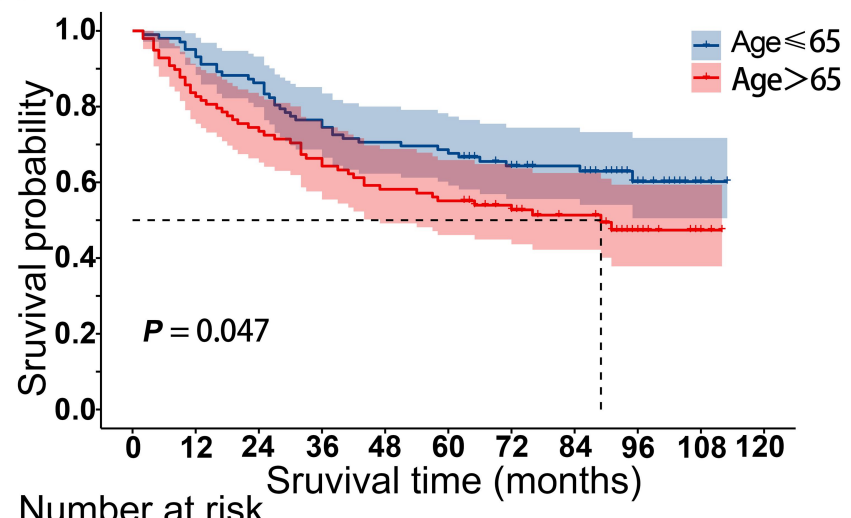

Number at risk

\begin{tabular}{|c|c|c|c|c|c|c|}
\hline 97 & 97 & & 72 & 70 & 54 & \\
\hline 82 & 327 & 65 & 57 & 54 & 44 & \\
\hline
\end{tabular}

\section{C}

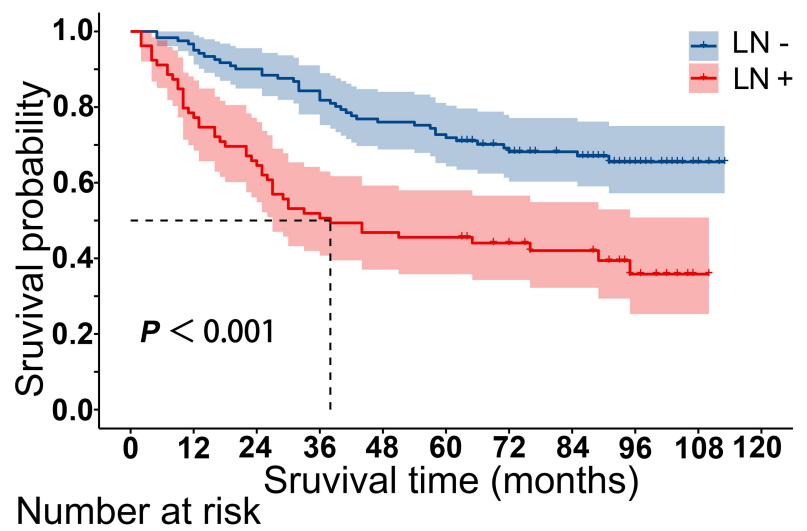

B

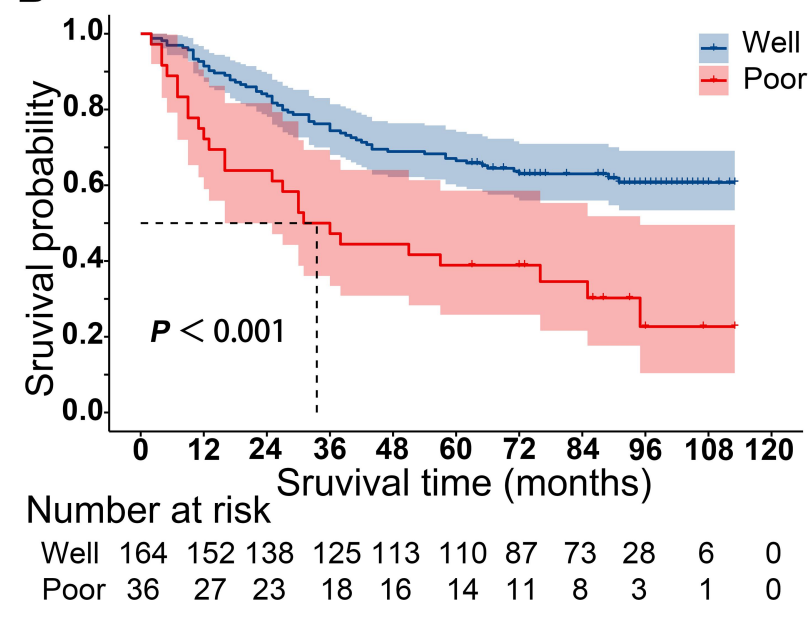

D

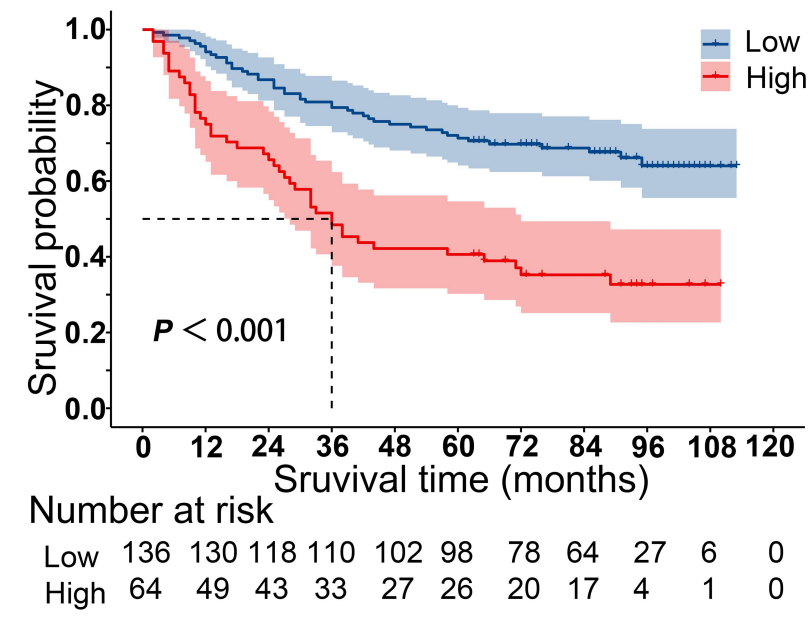

Figure 3 Kaplan-Meier curves for overall survival according to the (A) age; (B) differentiation; (C) lymph node metastasis; (D) TIPE expression level.

follow-up data were available. The expression levels of TIPE in the colon cancer samples and adjacent normal samples were assessed using immunohistochemistry. The expression levels of TIPE in colon cancer samples and adjacent normal samples were assessed using immunohistochemistry. TIPE immunostaining was predominantly

Table 5 Prognostic Value of TIPE Expression and Clinicopathological Factors for the Overall Survival of Colon Cancer Patients by Univariate and Multivariate Analyses with Cox Regression

\begin{tabular}{|l|l|l|l|l|l|l|}
\hline \multirow{2}{*}{ Variable } & \multicolumn{2}{l|}{ Univariate } & \multicolumn{2}{l|}{ Multivariate } \\
\cline { 2 - 6 } & HR & Cl (95\%) & P-value & HR & CI (95\%) & P-value \\
\hline Age (years): $>65$ vs $\leq 65$ & 1.529 & $1.001-2.336$ & 0.050 & 1.554 & $1.013-2.385$ & 0.043 \\
Gender: male vs female & 0.836 & $0.549-1.272$ & 0.403 & & \\
Differentiation: poor vs moderate and well & 2.547 & $1.598-4.059$ & $<0.001$ & 2.444 & $1.528-3.911$ \\
Lymphatic invasion: Yes vs no & 2.508 & $1.643-3.829$ & $<0.001$ & 1.999 & $1.288-3.103$ & $<0.001$ \\
Depth of invasion: T3+4 vs TI+2 & 6.378 & $0.888-45.812$ & 0.066 & & & \\
Tumor location: right vs left & 1.178 & $0.769-1.805$ & 0.452 & & & \\
Histological type: MC vs NMC & 1.105 & $0.633-1.929$ & 0.725 & & \\
TIPE expression: High vs Low & 2.751 & $1.803-4.197$ & $<0.001$ & 2.353 & $1.521-3.642$ \\
\hline
\end{tabular}

Abbreviations: HR, hazard ratio; MC, mucinous adenocarcinoma; NMC, non-mucinous adenocarcinoma. 
observed in the cytoplasm of carcinoma cells and rarely in the nucleus. In the present study, TIPE protein expression was significantly higher in colon cancer samples than in adjacent normal tissue samples. TIPE is considered a potential biomarker for colon cancer diagnosis, although the underlying mechanism leading to TIPE overexpression in human colon cancer is unclear. Future studies will involve next-generation sequencing and whole-genomerelated technologies to study genomic changes. ROC curve analysis showed that the IRS of TIPE had good predictive value for five-year survival rates and lymph node metastasis, especially among patients with RCC. A more accurate predictive model for outcomes could be established by combining TIPE expression and Dukes' staging system.

Currently, there is very limited information on the regulation of TIPE expression and its biological function in cancer cells. TIPE can modulate a variety of processes involved in cancer progression and metastasis. In vivo and in vitro experiments have confirmed that TIPE promotes the metastasis of colorectal cancer and breast cancer by regulating VEGFR2-mediated angiogenesis. ${ }^{16,31,35}$ TIPE overexpression is detected in human intestinal-type gastric adenocarcinoma and correlated with lymph node metastasis and poor patient prognosis. ${ }^{30}$ Previous studies also demonstrated that $\mathrm{pN} 0$ esophageal squamous cell carcinoma patients with TIPE overexpression have a higher risk of postoperative lymphatic recurrence. $^{29}$ Overexpression of TIPE was also detected in pancreatic cancer tumours, and the expression was correlated with tumour stage, lymph node metastasis, and epidermal growth factor receptor (EGFR) levels, contributing to disease progression. ${ }^{36}$ However, not all conclusions are consistent, and overexpression of TIPE was significantly correlated with good prognosis in HCC according to the RNA-seq data from The Cancer Genome Atlas (TCGA) database. $^{27}$

The association of TIPE with colon cancer has rarely been reported. In this study, we sought to determine the prognostic value and biological role of TIPE in patients with colon cancer. Clinicopathological features and colon cancer-associated prognostic factors were investigated to provide a reliable theoretical understanding of influencing factors in the prognosis of colon cancer. The present study confirmed that TIPE was overexpressed in primary colon cancer, which was correlated with Dukes' stage, lymph node metastasis and poor prognosis of colon cancer. TIPE expression was higher in Dukes' stage C tumours than in stage $A \& B$ tumours and was also higher in tumours with lymph node metastasis than in those without lymph node metastasis. These findings are in alignment with the findings of previous studies, which suggests that TIPE may serve as a molecular marker to predict cancer behaviour in individual patients..$^{17,34,37}$ Knockdown of TIPE expression has been shown to downregulate VEGFR2 expression, which results in the simultaneous inhibition of cell proliferation, cell migration and angiogenesis. ${ }^{35}$ It was also reported that knockdown of TIPE expression inhibits colon cancer cell proliferation and colony formation. ${ }^{38}$ The present findings indicate that TIPE may serve as a potential therapeutic target for colon cancer.

A couple of potential drawbacks of this study should be addressed. First, the present study had a relatively small sample size, and as a result, a larger sample size study is needed to confirm the reliability of the results. Second, as we used an IHC staining method, the results may be affected by interobserver heterogeneity. To circumvent this, we involved two experienced pathologists in the scoring process. However, subsequent in-depth research into its molecular mechanism is needed.

\section{Conclusion}

In conclusion, compared with that in adjacent healthy colon tissue, the expression level of TIPE in colon cancer tissue was higher, especially in RCC tissue. TIPE overexpression correlated with higher Dukes' stage, lymph node metastasis, RCC and shorter overall survival times. Moreover, multivariate analysis indicated that advanced age, Dukes' stage, lymph node metastases and TIPE overexpression were independent prognostic factors in colon cancer patients. ROC curve analysis showed that the IRS of TIPE had good predictive value for five-year survival rates and lymph node metastasis, especially among patients with RCC. TIPE may be useful as a molecular marker for RCC diagnosis and a drug target for RCC treatment.

\section{Disclosure}

The authors report no conflicts of interest in this work.

\section{References}

1. Keum NN, Giovannucci E. Global burden of colorectal cancer: emerging trends, risk factors and prevention strategies. Nat Rev Gastroenterol Hepatol. 2019;16(5). 
2. Siegel R, Miller K, Jemal A. Cancer statistics, 2019. CA Cancer J Clin. 2019;69(1):7-34. doi:10.3322/caac.21551

3. Siegel R, Miller K, Jemal A. Cancer statistics, 2020. CA Cancer $J$ Clin. 2020;70(1):7-30. doi:10.3322/caac.21590

4. Zhang L, Cao F, Zhang G, et al. Trends in and predictions of colorectal cancer incidence and mortality in China from 1990 to 2025. Front Oncol. 2019;9:98. doi:10.3389/fonc.2019.00098

5. Siegel R, Miller K, Goding Sauer A, et al. Colorectal cancer statistics, 2020. CA Cancer J Clin. 2020;70(3):145-164. doi:10.3322/ caac. 21601

6. Qiu M, Pan W, Lin J, et al. Comparison of survival between right-sided and left-sided colon cancer in different situations. Cancer Med. 2018;7(4):1141-1150. doi:10.1002/cam4.1401

7. Imperial $\mathrm{R}$, Ahmed $\mathrm{Z}$, Toor $\mathrm{O}$, et al. Comparative proteogenomic analysis of right-sided colon cancer, left-sided colon cancer and rectal cancer reveals distinct mutational profiles. Mol Cancer. 2018;17 (1):177. doi:10.1186/s12943-018-0923-9

8. Petrelli F, Tomasello G, Borgonovo K, et al. Prognostic survival associated with left-sided vs right-sided colon cancer: a systematic review and meta-analysis. JAMA oncol. 2017;3(2):211-219. doi:10.1001/jamaoncol.2016.4227

9. Baig N, Li Z, Lu J, et al. Clinical significance and comparison of flotillin 1 expression in left and right colon cancer. Oncol Lett. 2019;18(2):997-1004. doi:10.3892/ol.2019.10401

10. Wang Q, Zhang M, Shi C, et al. High Oct4 predicted worse prognosis of right-sided colon cancer patients. Future Oncol. 2018;14 (22):2279-2291. doi:10.2217/fon-2018-0046

11. Hussain M, Waqas O, Hassan U, et al. Right-sided and left-sided colon cancers are two distinct disease entities: an analysis of 200 cases in Pakistan. Asian Pac J Cancer Prev. 2016;17(5):2545-2548.

12. Yaeger R, Chatila W, Lipsyc M, et al. Clinical sequencing defines the genomic landscape of metastatic colorectal cancer. Cancer Cell. 2018;33(1):125-136.e3. doi:10.1016/j.ccell.2017.12.004

13. Nawa T, Kato J, Kawamoto H, et al. Differences between right- and left-sided colon cancer in patient characteristics, cancer morphology and histology. $J$ Gastroenterol Hepatol. 2008;23(3):418-423. doi:10.1111/j.1440-1746.2007.04923.x

14. Karim S, Brennan K, Nanji S, Berry S, Booth C. Association between prognosis and tumor laterality in early-stage colon cancer. JAMA Oncol. 2017;3(10):1386-1392. doi:10.1001/jamaoncol.2017.1016

15. Patel S, Wang F, Whiteside T, Kasid U. Identification of seven differentially displayed transcripts in human primary and matched metastatic head and neck squamous cell carcinoma cell lines: implications in metastasis and/or radiation response. Oral Oncol. 1997;33 (3):197-203. doi:10.1016/s0964-1955(96)00065-6

16. Kumar D, Gokhale P, Broustas C, Chakravarty D, Ahmad I, Kasid U. Expression of SCC-S2, an antiapoptotic molecule, correlates with enhanced proliferation and tumorigenicity of MDA-MB 435 cells. Oncogene. 2004;23(2):612-616. doi:10.1038/sj.onc.1207123

17. Niture S, Dong X, Arthur E, et al. Oncogenic role of tumor necrosis factor $\alpha$-induced protein 8 (TNFAIP8). Cells. 2018;8(1):9. doi:10.3390/cells8010009

18. Niture S, Gyamfi M, Lin M, et al. TNFAIP8 regulates autophagy, cell steatosis, and promotes hepatocellular carcinoma cell proliferation. Cell Death Dis. 2020;11(3):178. doi:10.1038/s41419-020-2369-4

19. Xiao Y, Huang S, Qiu F, et al. Tumor necrosis factor $\alpha$-induced protein 1 as a novel tumor suppressor through selective downregulation of CSNK2B blocks nuclear factor- $\mathrm{KB}$ activation in hepatocellular carcinoma. EBioMedicine. 2020;51:102603. doi:10.1016/j. ebiom.2019.102603

20. Li Z, Zhang W, Li Y, et al. TIPE2 acts as a biomarker for GIST risk category and suppresses the viability and invasiveness of GIST cells. Cell Biosci. 2018;8(1):62. doi:10.1186/s13578-018-0261-z

21. Fayngerts S, Wu J, Oxley C, et al. TIPE3 is the transfer protein of lipid second messengers that promote cancer. Cancer Cell. 2014;26 (4):465-478. doi:10.1016/j.ccr.2014.07.025
22. Zhong M, Zhu M, Liu Y, et al. TNFAIP8 promotes the migration of clear cell renal cell carcinoma by regulating the EMT. $J$ Cancer. 2020;11(10):3061-3071. doi:10.7150/jca.40191

23. Moniz L, Vanhaesebroeck B. A new TIPE of phosphoinositide regulator in cancer. Cancer Cell. 2014;26(4):443-444. doi:10.1016/j. ccell.2014.09.017

24. Zhang L, Liu R, Luan Y, Yao Y. Tumor necrosis factor- $\alpha$ induced protein 8: pathophysiology, clinical significance, and regulatory mechanism. Int J Biol Sci. 2018;14(4):398-405. doi:10.7150/ ijbs. 23268

25. Weiser M. AJCC 8th Edition: colorectal Cancer. Ann Surg Oncol. 2018;25(6):1454-1455. doi:10.1245/s10434-018-6462-1

26. Araújo R, Lira G, Vilaça J, et al. Prognostic and diagnostic implications of MMP-2, MMP-9, and VEGF- $\alpha$ expressions in colorectal cancer. Pathol Res Pract. 2015;211(1):71-77. doi:10.1016/j. prp.2014.09.007

27. Padmavathi G, Banik K, Monisha J, et al. Novel tumor necrosis factor- $\alpha$ induced protein eight (TNFAIP8/TIPE) family: functions and downstream targets involved in cancer progression. Cancer Lett. 2018;432:260-271. doi:10.1016/j.canlet.2018.06.017

28. Goldsmith J, Fayngerts S, Chen Y. Regulation of inflammation and tumorigenesis by the TIPE family of phospholipid transfer proteins. Cell Mol Immunol. 2017;14(6):482-487. doi:10.1038/cmi.2017.4

29. Sun Z, Liu X, Song J, et al. TNFAIP8 overexpression: a potential predictor of lymphatic metastatic recurrence in $\mathrm{pN} 0$ esophageal squamous cell carcinoma after Ivor Lewis esophagectomy. Tumour Biol. 2016;37(8):10923-10934. doi:10.1007/s13277-016-4978-1

30. Yang M, Zhao Q, Wang X, et al. TNFAIP8 overexpression is associated with lymph node metastasis and poor prognosis in intestinal-type gastric adenocarcinoma. Histopathology. 2014;65 (4):517-526. doi:10.1111/his.12413

31. Zhong M, Li N, Qiu X, et al. TIPE regulates VEGFR2 expression and promotes angiogenesis in colorectal cancer. Int J Biol Sci. 2020;16 (2):272-283. doi:10.7150/ijbs.37906

32. Yang C, Xu W, Meng X, Zhou S, Zhang M, Cui D. SCC-S2 facilitates tumor proliferation and invasion via activating Wnt signaling and depressing hippo signaling in colorectal cancer cells and predicts poor prognosis of patients. J Histochem Cytochem. 2019;67 (1):65-75. doi:10.1369/0022155418799957

33. Zhong M, Chen Z, Yan Y, et al. Expression of TIPE family members in human colorectal cancer. Oncol Lett. 2021;21(2):118. doi:10.3892/ ol.2020.12379

34. Lowe J, Nguyen T, Grimm S, et al. The novel p53 target TNFAIP8 variant 2 is increased in cancer and offsets p53-dependent tumor suppression. Cell Death Differ. 2017;24(1):181-191. doi:10.1038/ cdd.2016.130

35. Zhang C, Chakravarty D, Sakabe I, et al. Role of SCC-S2 in experimental metastasis and modulation of VEGFR-2, MMP-1, and MMP-9 expression. Mol Ther. 2006;13(5):947-955. doi:10.1016/j. ymthe.2005.11.020

36. Liu K, Qin C, Wang Z, Liu S, Cui X, Zhang D. Expression of tumor necrosis factor-alpha-induced protein 8 in pancreas tissues and its correlation with epithelial growth factor receptor levels. Asian Pac J Cancer Prev. 2012;13(3):847-850. doi:10.7314/ apjcp.2012.13.3.847

37. Shen H, Yang J, Huang Q, et al. Different treatment strategies and molecular features between right-sided and left-sided colon cancers. World J Gastroenterol. 2015;21(21):6470-6478. doi:10.3748/wjg. v21.i21.6470

38. Miao Z, Zhao T, Wang Z, et al. SCC-S2 is overexpressed in colon cancers and regulates cell proliferation. Tumour Biol. 2012;33 (6):2099-2106. doi:10.1007/s13277-012-0469-1 


\section{Publish your work in this journal}

Cancer Management and Research is an international, peer-reviewed open access journal focusing on cancer research and the optimal use of preventative and integrated treatment interventions to achieve improved outcomes, enhanced survival and quality of life for the cancer patient.
The manuscript management system is completely online and includes a very quick and fair peer-review system, which is all easy to use. Visit http://www.dovepress.com/testimonials.php to read real quotes from published authors.

Submit your manuscript here: https://www.dovepress.com/cancer-management-and-research-journal 